\title{
GLAD!
}

Revue sur le langage, le genre, les sexualités

$10 \mid 2021$

Varia

\section{Sous la conduite d'Hermès, aux carrefours de la traduction et du féminisme}

At the Crossroads of Translation and Feminism under the Guidance of Hermes

Jane Elisabeth Wilhelm

\section{(2) OpenEdition}

\section{Journals}

Édition électronique

URL : https://journals.openedition.org/glad/2883

DOI : $10.4000 /$ glad.2883

ISSN : 2551-0819

Éditeur

Association GSL

Référence électronique

Jane Elisabeth Wilhelm, «Sous la conduite d'Hermès, aux carrefours de la traduction et du

féminisme », GLAD! [En ligne], 10 | 2021, mis en ligne le 15 juillet 2021, consulté le 26 juillet 2021. URL : http://journals.openedition.org/glad/2883; DOI : https://doi.org/10.4000/glad.2883

Ce document a été généré automatiquement le 26 juillet 2021.

\section{(c) (†) $\ominus$}

La revue GLAD! est mise à disposition selon les termes de la Licence Creative Commons Attribution -

Pas d'Utilisation Commerciale - Pas de Modification 4.0 International. 


\section{Sous la conduite d'Hermès, aux carrefours de la traduction et du féminisme}

At the Crossroads of Translation and Feminism under the Guidance of Hermes

Jane Elisabeth Wilhelm

\section{Introduction}

Nous souhaitons examiner ici quelques-uns des lieux de convergence entre traduction, herméneutique et féminisme. Si les termes de traduction et de féminisme peuvent paraitre clairs au premier abord, il n'en va pas de même pour l'herméneutique qui appelle d'entrée de jeu une définition ${ }^{1}$. Pourtant, l'abondance des métaphores dans les différentes langues suggère que la traduction est difficile à définir et résiste à la conceptualisation ${ }^{2}$. Si tout le monde sait ce qu'est traduire, affirme Jean-René Ladmiral, paradoxalement, le concept de traduction est difficile à définir (Ladmiral 2015: 91). Ne faudrait-il pas toutefois en préambule tenter de donner une définition possible de la traduction? Dans les discours théoriques, il semblerait que ce soit en termes d'équivalence (comme l'a fait Eugene Nida) ou d'identité que l'on a le plus souvent pensé la traduction, mais ces définitions se révèleraient être insatisfaisantes selon Ladmiral. La difficulté à définir le concept de traduction est donc l'indice, selon lui, qu'elle renvoie à des enjeux importants que l'on ne décèle pas à première vue et que la traduction recèle « une dimension anthropologique fondamentale " (Ladmiral 2015 : 93, en italique dans le texte). Paul Ricœur évoque lui aussi toute la difficulté théorique que pose la traduction («théoriquement incompréhensible, mais effectivement praticable ») dans une définition présentée sous la forme de ce qu'il nomme un "paradoxe» et un «dilemme»: «une bonne traduction ne peut viser qu'à une équivalence présumée, non fondée dans une identité de sens démontrable. Une équivalence sans identité. » ${ }^{3}$ La traduction pour lui, comme pour Hans-Georg Gadamer, est le modèle ou le « paradigme » de l'herméneutique. 
2 Florence Rochefort, dans son Histoire mondiale des féminismes, a choisi de présenter le féminisme au pluriel. Le mot, d'abord employé péjorativement et qui est entré comme par effraction dans le langage au 19e siècle, a souvent gardé même dans son acception moderne, comme elle le rappelle, « un sens subversif et dérangeant " (2018: 7). Maud Navarre, dans l'éditorial intitulé «La force d'une idée » du numéro de la revue Sciences Humaines consacré à "La grande histoire du féminisme», soutient de plus qu'en s'inscrivant dans les courants de pensée et les doctrines politiques de son temps, «le féminisme se veut pluriel dès son commencement " (2021). Il est à l'évidence que le mouvement d'émancipation des femmes au niveau international et dans ses différentes déclinaisons, qui représente le mouvement social le plus important de l'époque moderne, est apparu en même temps que se développait dans les pays occidentaux l'idée de démocratie. Nous développerons par la suite le rapport du politique avec l'enjeu herméneutique de la pluralité de l'interprétation et la question de la femme au cœur de la démocratie.

3 Avant d'examiner les points de jonction entre traduction, féminisme et herméneutique, en nous appuyant plus particulèrement sur le " paradigme de la traduction » de Paul Ricœur et son concept d'hospitalité langagière, nous allons expliciter plus spécifiquement les rapports que la traduction entretient avec l'herméneutique.

\section{Herméneutique et théorie de la traduction}

«L'herméneutique », rappelle Jean Starobinski dans l'Avant-propos à l'Herméneutique de Friedrich Schleiermacher, "peut se définir comme l'art de comprendre et d'interpréter » (1987: 5, en italique dans le texte). L'étymologie du mot hermeneuein en grec, écrit-il, signifie "exprimer, expliquer, ou traduire d'une langue à l'autre». Le trait commun à ces trois sens, écrit-il encore, est la notion de "médiation en vue de produire un message intelligible». Dans les emplois de la langue grecque, nous dit-il, le message a un caractère de vérité et d'autorité, car interpréter peut signifier déclarer la volonté des dieux (Starobinski 1987 : 5, en italique dans le texte). Jean Grondin écrit, lui aussi, qu'on reconnait largement trois significations au verbe grec: «exprimer (énoncer, dire), interpréter (expliquer) et traduire (ou servir d'interprète) » (Grondin 1993 : 6). Dans l'histoire de l'herméneutique, écrit Gadamer (1976), le modèle est la compétence augurale de l'herméneute sachant interpréter la volonté divine et le langage de l'oracle, qui est le plus souvent obscur ou ambigu ${ }^{4}$. Et c'est ici, pour Starobinski, qu'entre en scène Hermès, "divinité messagère, patron des interprètes, guide des âmes, maître des échanges, régnant sur les carrefours du vrai et du faux " (Starobinski $1987: 5$ ). On pourrait rajouter qu'Hermès, messager des dieux et patron de l'herméneutique, est aussi donneur de chance, inventeur des poids et des mesures, gardien des routes et des carrefours, dieu des voyageurs, des commerçants et des... voleurs!

5 L'herméneutique philosophique contemporaine - représentée en France par Paul Ricœur, en Allemagne par Martin Heidegger, Hans-Georg Gadamer ou Jürgen Habermas, parmi d'autres - en déplaçant la question herméneutique vers le lecteur, la production de sens du texte comme procès dynamique, ainsi que l'espace de négociation entre l'auteur et le lecteur, cherche à prendre la mesure de l'espace infini de l'interprétation qui, sous l'égide du dieu Hermès, s'ouvre à nous. 
6 La théorie de la traduction, selon Antoine Berman, aurait pour fondement deux grands courants, la linguistique et l'herméneutique (Berman 1989). Si la linguistique, comme l'explique Mona Baker, a été la discipline qui a servi de modèle à l'étude de la traduction au début des années 1950 et durant les années 1960, la voie royale de l'herméneutique est au fondement de l'interprétation des textes sacrés des religions monothéistes ${ }^{5}$. Andrew Chesterman distingue plus généralement deux périodes historiques qu'il nomme "the Linguistic stage " et "the Logos stage ", la seconde caractérisée selon lui par une approche herméneutique (Chesterman 1997 : 42-43). Si elle est présente en critique littéraire et en particulier chez Jean Starobinski ${ }^{6}$, la dimension herméneutique n'apparaît pourtant pas clairement chez les théoriciens de la traductologie de langue française, à l'exception d'Antoine Berman ou plus récemment de manière explicite chez Jean-René Ladmiral, par ailleurs élève de Ricœur. L'anthropologie philosophique de Ricœur peut alors se concevoir plus largement, selon Domenico Jervolino, comme une traversée du langage qui partirait de l'herméneutique des symboles vers le texte pour aboutir au «paradigme de la traduction " (Jervolino 2004 : 659-668). Rappelons encore que Friedrich Schleiermacher, qui était théologien, philosophe et traducteur et à qui l'on doit la naissance de l'herméneutique moderne, est aussi considéré comme une figure importante pour les théoriciens de la traduction 7 . En effet, Lawrence Venuti, Douglas Robinson, Anthony Pym ou Antoine Berman ont souligné le rôle inaugural du discours de Schleiermacher à l'Académie des sciences de Berlin de 1813 intitulé Des différentes méthodes du traduire (Schleiermacher 1999). Berman rappelle en outre la critique radicale que fait Schleiermacher de ce qu'il appelle «la traduction ethnocentrique et hypertextuelle» sur laquelle nous reviendrons ultérieurement (Berman 1999 : 78).

7 En retraçant les différentes étapes de l'histoire de l'herméneutique à partir de l'interprétation de la volonté des dieux, puis d'une science de l'interprétation des textes qui se voulait essentiellement technique ou normative, à l'herméneutique philosophique contemporaine, qui relève de l'ordre de la connaissance et qui est aussi une lecture du monde, on peut constater que le texte constitue la dimension essentielle de l'herméneutique. Le point de rencontre de la tradition herméneutique et de la théorie de la traduction s'articule ainsi autour de la lecture du texte, qui appelle donc l'acte d'interprétation. La situation commune à l'art de l'interprétation et à la traduction, qui lui est nécessairement liée pour Gadamer ou Ricœur, et dont part l'herméneutique de Schleiermacher, est énoncée dans une formule restée célèbre: "L'herméneutique repose sur le fait de la non-compréhension du discours » (Schleiermacher $1987: 73$ ).

8 Si Hermès est le maître des échanges, quels seraient dès lors les lieux de convergence entre traduction, herméneutique et féminisme? D'emblée, nous voyons les points de rencontre suivants que nous développerons ici: la question du sens, celle de l'invisibilité et de la justice, ainsi que la confrontation avec l'altérité de l'étranger. Nous avons déjà traité en détail de la question du pouvoir et de l'autorité en lien avec la traduction féministe dans notre article intitulé « Anthropologie des lectures féministes de la traduction " (Wilhelm 2014). Nous y reviendrons dans la partie qui traitera de l'invisibilisation des femmes en traduction et de la rencontre entre traductologie et pensée féministe.

9 La question de la traduction comme paradigme ou modèle de l'herméneutique a été largement développée par Hans-Georg Gadamer et Paul Ricœur dans leur œuvre. Par 
ailleurs, des féministes se sont également intéressées à la pensée de ces deux philosophes et deux ouvrages en particulier doivent retenir toute notre attention: Feminist Interpretations of Hans-Georg Gadamer (Code 2003) et Feminist Explorations of Paul Ricœur's Philosophy (Halsema \& Henriques 2016). Si l'on a pu analyser la traduction au sein de l'herméneutique à la suite de Gadamer et de Ricœur dans différentes études, ou encore conjuguer herméneutique et féminisme comme c'est le cas dans les deux recueils cités ci-dessus, l'originalité de notre démarche est d'aborder ici les trois dimensions mentionnées précédemment dans l'introduction, à savoir traduction, herméneutique et féminisme.

\section{La question du sens}

\section{L'accession de l'herméneutique à l'universalité}

10 L'herméneutique est la discipline qui traite de manière privilégiée de la question du sens et dont Michel Foucault donne la définition suivante dans Les Mots et les choses: "l'ensemble des connaissances et des techniques qui permettent de faire parler les signes et de découvrir leur sens » (Foucault $1966: 44)$ ). On peut se demander comment la discipline qui avait pour objet l'interprétation, qui s'est constituée depuis la Grèce antique comme l'ensemble des règles présidant à l'explication des textes sacrés, juridiques ou littéraires, à savoir un art technique, a pu devenir une théorie philosophique. S'il revient à l'exégèse d'instituer l'herméneutique en tant que discipline auxiliaire de la théologie, sa longue histoire est inaugurée par la rhétorique qui, en s'attachant à la production des discours, démontre les règles discursives qui commandent le passage d'une figure à une autre. La lecture herméneutique, en assurant le passage d'un état de langue à un autre, ne s'intéresse pas à la production des discours, comme la rhétorique, mais au souci de leur compréhension ${ }^{8}$. Que faut-il interpréter dans les textes sacrés et pour quelle raison? La critique appliquée aux textes, explique Jean Molino, révèle que « le langage n'est pas transparent, pas plus transparent que le monde auquel il renvoie» (Molino 1985: 88,93) et que nous ne saurions en rester à l'illusion trompeuse d'une signification immédiate. Un texte, qu'il soit révélé ou non, peut avoir plusieurs sens et c'est notamment le cas de la Bible ${ }^{9}$ : on connaît la célèbre formule de Grégoire le Grand "Scriptura cum legentibus crescit " (« l'Écriture croît avec ceux qui la lisent ») ${ }^{10}$. La Réforme fit éclater le conflit des interprétations divergentes entre l'exégèse catholique et protestante. L'exégèse catholique situe la lecture des Écritures au sein d'une tradition où le sens spirituel est conforme au dogme. Les réformateurs protestants, par contre, en critiquant le rôle joué par la tradition de l'Église dans l'interprétation des Écritures, privilégieront le contact direct avec la Bible pour en dégager un sens littéral ou historique qui est actualisé. L'activité herméneutique a ainsi joué un rôle essentiel dans la lecture de la Bible et toutes ses traductions dans les différentes langues au cours des âges.

11 Friedrich Schleiermacher, qui était théologien, se proposa de donner des fondements théoriques et une dimension universelle à l'herméneutique en changeant sa tâche. En privilégiant la question du sens, il fonde l'herméneutique moderne sur l'acte de la compréhension au cœur de l'interprétation. L'herméneutique de Schleiermacher, introduisant la dichotomie de l'objectif et du subjectif, se base sur l'expérience de la vie, le dialogue et le problème de l'incompréhension ou du malentendu. L'art de la 
compréhension et de l'explication s'émancipe alors des disciplines interprétatives, à savoir l'exégèse, la philologie, le droit ou l'histoire, dont il n'était jusqu'alors que le simple auxiliaire... Heidegger reprendra la tradition herméneutique issue de Schleiermacher en dépouillant la compréhension de son caractère purement épistémique pour lui donner un fondement plus universel, celui du monde vécu: l'élucidation de la structure d'anticipation de la compréhension signifie dès lors que l'existence humaine elle-même se caractérise par sa dimension interprétative ( $c f$. Grondin 1993 : 132). La description phénoménologique du cercle herméneutique par Heidegger met ainsi en lumière la structure d'anticipation et le caractère de projet de toute compréhension de l'être humain dans le monde.

Si l'herméneutique, constituée en réflexion sur les conditions du savoir revendique le statut de philosophie première («fondamentale et universelle» selon Hans-Georg Gadamer), c'est en raison de l'universalité de la question de l'interprétation qui s'impose aujourd'hui comme l'un des horizons de la pensée contemporaine ${ }^{11}$. L'universalité de l'ordre interprétatif, incarné dans le langage et considéré comme la dimension fondamentale de l'existence humaine, trouve son champ d'application dans tous les domaines de la culture, y compris la pensée féministe. Dans cette perspective, Georgia Warnke rappelle que si les féministes se tournent souvent vers la philosophie postmoderne pour trouver un cadre conceptuel au sein duquel traiter la question de la différence, l'herméneutique philosophique dans sa dimension politique est peut-être plus en mesure d'appuyer les arguments féministes relatifs à la dimension interprétative de la différence. L'herméneutique, écrit-elle encore, nous permet aussi de reconnaitre le caractère partial de toute compréhension (Warnke 1993) ${ }^{12}$. Certains concepts de Gadamer peuvent s'avérer particulièrement pertinents pour une épistémologie féministe, en particulier ceux de "préjugé» (Vor-Urteil), de "précompréhension ", de situation historique ou de "fusion des horizons ", comme les contributions au recueil intitulé Feminist Interpretations of Gadamer peuvent l'illustrer à juste titre (cf. Code 2003). Dans son article, Alison Scott Baumann suggère de définir le féminisme comme étant le propre de ces hommes et de ces femmes "who seek to challenge the subjugation of women, draw attention to implicit bias (the undeclared privileging of one group over another), and to stereotype threat (perception of characteristics that must be retained in order to retain membership of one's group) and propose positive alternatives» (Scott-Baumann 2016:39). Cette définition a le mérite de souligner le rôle de la précompréhension («implicit bias»), cette structure d'anticipation du comprendre décrite par Heidegger, et celle des stéréotypes avec la question des métaphores sexistes et sexuelles en traduction que nous avons abordée dans deux précédents articles (Chamberlain ${ }^{13} 2004$ [1988]; Wilhelm 2014 et Wilhelm 2019).

\section{L'enjeu herméneutique : pluralité de l'interprétation, démocratie et féminisme}

En soulignant la dimension éthique et sociale de l'enjeu herméneutique, Michel Jeanneret soutient que la question de la pluralité de l'interprétation est à mettre en lien avec la liberté de pensée. Il cite dans ce contexte Alain Berthoz, professeur de physiologie et de sciences cognitives au Collège de France, pour qui l'acceptation de la pensée d'autrui et la défense de l'interprétation plurielle inspirent la tolérance et le respect démocratique (Jeanneret 2015). La reconnaissance d'un partenariat équitable 
entre les genres apparaît depuis des décennies comme l'une des conditions essentielles de la démocratie, du développement durable et de la paix selon les rapports des différentes agences de l'Organisation des Nations Unies (ONU). L'avocate et militante féministe Gisèle Halimi rappelle que "l'avancée des femmes a toujours renforcé la démocratie " et que "c'est seulement en mettant au centre du débat la question de l'identité sexuée, en reconnaissant la dualité du genre humain, que la démocratie peut se construire. À défaut, le corps politique est mutilé.» (Halimi 1994) Pour la sociologue Eva Illouz, le féminisme représente « rien de moins que la deuxième grande étape du processus de démocratisation des sociétés européennes, qui commença au XVIIIe siècle. Aussi longtemps que les femmes en étaient exclues, la démocratie était restée un projet inachevé, incohérent et tronqué, crée par des hommes pour des hommes " (Illouz 2020). Dans un entretien avec l'historienne Michelle Perrot, la philosophe et psychologue Carol Gilligan, à l'origine de la théorie du «care ", affirme à son tour le lien entre féminisme et démocratie. Le féminisme, à ses yeux, est « l'instauration d'une démocratie égalitaire qui s'arrache au système patriarcal », et cela "constitue le plus important mouvement de libération des individus, hommes et femmes» (Radier 2019: 72). Toutefois, avant de pouvoir s'extraire du patriarcat, dont elle rappelle que certaines disciplines comme l'anthropologie «ont mis en évidence combien nous tendons à interpréter cette culture patriarcale comme un état naturel» (Radier 2019: 70-71), encore faut-il pouvoir en démonter les mécanismes et les «préjugés » au sens herméneutique du terme. La lutte des femmes pour l'égalité, l'accession au droit de vote, aux droits civils et politiques, s'est accompagnée d'un combat contre les préjugés sexistes et ses représentations illustrées notamment par les stéréotypes négatifs liés au féminin et le sens commun misogyne. L'enjeu de la compréhension des stéréotypes et des représentations de la femme dans la société, tels qu'ils sont illustrés dans les discours sur la traduction (Chamberlain 2004 [1988]; Wilhelm 2014), se révèlent être essentiels dans une perspective d'analyse féministe, non seulement pour mettre en lumière les ressorts du l'ordre patriarcal, mais plus largement pour l'avenir de la démocratie selon le sociologue Dominique Wolton (Wolton 2019: 18). Dans une perspective féministe, il s'agit bien pour les femmes de "réinventer le pouvoir", comme l'indique le sous-titre du livre de Marie-Cécile Naves, La démocratie féministe. L'imaginaire démocratique, à ses yeux, est ouvert : le féminisme permet d'enrichir la démocratie et d'accroître les libertés humaines. Le féminisme, écrit-elle encore, permet d'« aider à penser par soi-même, à développer son imagination, à faire confiance à sa capacité d'interprétation de la réalité, à faire évoluer les relations interpersonnelles, les normes et les représentations sociales » (Naves 2020 : 272, c'est nous qui soulignons).

\section{La question du sens au cœur des sciences humaines : le paradigme de la traduction}

14 En s'attachant à l'examen de la représentation des objets ou des réalités symboliques, les lettres et les sciences humaines et sociales, au sein desquelles on trouve la traductologie et les études de genre, s'inscrivent dans l'horizon de la question du sens posée magistralement par Schleiermacher au début du XIXe siècle. Jean Molino rappelle que l'herméneutique, "l'art d'interpréter les signes" a dès ses origines rassemblé au cours de sa longue histoire un vaste savoir autour de cette question centrale, qu'est-ce qu'interpréter? (Molino 1985: 76). L'herméneutique est ainsi essentielle aux sciences humaines et sociales, que ce soit pour la constitution de leur 
objet, comme pour leur réflexivité. Aussi Silja Freudenberger écrit-elle que les épistémologies féministes doivent à Gadamer l'idée de l'importance fondamentale de la dimension herméneutique de toute connaissance (Freudenberger $2003: 281$ ). Dans cet horizon, le philosophe canadien Charles Taylor, qui engage une réflexion sur les conditions du savoir interprétatif et citant Gadamer, Ricœur et Habermas à l'appui, rappelle la dimension herméneutique des sciences humaines depuis Dilthey et le fait que l'interprétation est essentielle à toute explication (Taylor 2011 : 79). Susan Hekman, pour sa part, affirme que dès ses débuts, le féminisme a été une protestation contre le sens attribué à « la femme » et l'argument selon lequel ce sens devait être changé, ainsi que la société (Hekman 2003). Dans une perspective gadamérienne, écrit-elle encore, on pourrait dire que ce vers quoi tend le mouvement féministe depuis son commencement est la suspension des "préjugés » misogynes et la mise à jour des faux préjugés du patriarcat qui constituent l'infériorité de la femme (cf. Hekman 2003, 197-198; Gilligan et Snider 2019). La compréhension telle qu'elle s'applique aux sciences humaines et sociales, ainsi qu'aux questions féministes, n'a donc pas juste une dimension épistémique, car nous savons depuis Heidegger qu'elle s'inscrit plus fondamentalement dans le monde vécu et qu'elle est aussi explicitation de l'existence. L'herméneutique, par conséquent, s'attache à la lecture du monde. L'exercice de la compréhension quotidienne, selon Heidegger, s'accomplit de manière souvent implicite, comme mode d'être et dans une dimension pré-langagière. Aussi, dans la lutte des femmes pour la reconnaissance et l'égalité, la question du sens ou de la signification a-t-elle d'abord la dimension existentielle du vécu, celle de l'expérience de la discrimination et de l'injustice dans l'ordre social qui appelle dès lors une réponse politique, le mot grec polis renvoyant au vivre ensemble dans la cité.

15 Si la question du politique est à mettre en lien avec la pluralité de l'interprétation, la démocratie et l'avancée du féminisme, comme nous l'avons vu, il existe une dimension plus fondamentale encore qui informe à la fois la pensée féministe, la traduction (cf. Ladmiral cité précédemment) et l'herméneutique (cf. Molino 1985: 74-79): la perspective anthropologique. Dans un entretien accordé au Nouvel Observateur en décembre 2013, Judith Butler explique qu'elle n'a pas inventé les "études de genre » (gender studies), car le concept de "genre », nous dit-elle, était utilisé en sociologie et en anthropologie depuis les années 1960 (Butler 2013) ${ }^{14}$. Dans le champ de l'anthropologie féministe, citons en particulier les travaux de l'anthropologue et militante féministe américaine Gayle Rubin, qui a élaboré le concept de système de sexe/genre dans Marché aux femmes (The Traffic in Women), et ceux de l'anthropologue et féministe française Nicole-Claude Mathieu. Rappelons encore que pour l'ethnologue féministe Françoise Héritier, l'inégalité entre les sexes aurait été instaurée dès les origines à partir d'une interprétation discriminatoire de la différence biologique qui se révèle notamment dans la procréation (Héritier 2002; Wilhelm 2014). Le travail de l'anthropologue étudiant les relations sociales incarnées dans des formes symboliques est bien un travail d'interprétation, et l'anthropologie, tout comme la traduction, a un lien avec la question de l'étranger. Charles Taylor, examinant le cas particulier de la science politique, mais ceci vaut aussi pour l'ensemble des sciences humaines et sociales, distingue ainsi la dimension épistémique de l'interprétation, avec la signification linguistique, de la question ontologique de l'interprétation liée à ce qu'il appelle la "signification expérientielle» (Taylor 2011: 91) représentée par l'ensemble des pratiques sociales qui soutiennent des structures et des relations hiérarchiques de pouvoir. 
16 Ricœur, dont l'œuvre permet le dialogue avec la philosophie politique et notamment celle de Taylor, rappelle la pluralité de la condition humaine dans toutes les dimensions d'existence et dont «la diversité des langues est la manifestation la plus troublante » (Ricœur $2004: 58 ; 2016: 41$ ). Pour Ricœur, le terme de «traduction » peut être compris au sens linguistique strict, mais aussi "comme synonyme de l'interprétation de tout ensemble signifiant à l'intérieur de la même communauté linguistique " (Ricœur 2004: 21; 2016: 13). En créant un passage entre les langues, selon Barbara Cassin, la traduction «se situe d'emblée dans la dimension du politique : il y va de l'articulation d'une pluralité différenciée ». Le divers ${ }^{15}$, écrit-elle, « est mis en œuvre dans une pratique du commun ». Et elle affirme encore qu' « en tant que savoirfaire avec les différences ", la traduction peut constituer «le nouveau paradigme des sciences humaines» (Cassin 2014: 17). Cette définition d'un savoir-faire avec les différences qui caractérise la traduction paraît particulièrement bien s'appliquer aux études de genre et au cadre conceptuel au sein duquel traiter la prise en compte de «la pensée de la différence sexuelle », selon l'expression d'Hélène Cixous. Cette dernière évoque, comme Ricœur, la pluralité humaine et la nécessité de l'exercice d'hospitalité «à la multiplicité et aux diverses différences» dans le contexte de la démocratie: « Tout ce qui semble s'exercer comme idéologie dominante produit un effet d'exclusion évidemment. Or il faut toujours pouvoir ouvrir aux expressions des minorités. C'est le grand défi de la démocratie. La réalité humaine, c'est du pluriel, c'est du mélange, c'est de la combinaison de parties qui sont des touts ${ }^{16}$.»

\section{Le malentendu suscite l'interprétation}

17 Le propre de la tradition herméneutique, rappelle Ricœur, est d'aborder le problème de la compréhension à partir du problème du malentendu ou de la non-compréhension qui, "selon Schleiermacher, suscite l'interprétation, dont l'herméneutique veut faire la théorie $»^{17}$. Barbara Cassin rappelle, elle aussi, le problème de la non-compréhension au fondement de l'herméneutique de Schleiermacher ${ }^{18}$. Le recours à l'exercice d'interprétation s'impose à partir du moment où la compréhension se heurte à des difficultés et que l'on prend conscience du malentendu: l'interprète cherche alors à rendre compréhensible ce qui est étranger en s'efforçant d'interpréter les signes pouvant provoquer le malentendu. En retraçant les différentes étapes de l'histoire de l'herméneutique, on observe que les concepts de "propre», de "familiarité ", de «distance » ou d'«éloignement " reviennent avec insistance autour du thème de l'«étranger ». Ainsi Hans-Georg Gadamer écrit-il que la tâche de l'herméneutique se fonde sur "une polarité entre familiarité et étrangeté " (Gadamer 1976: 135). La traduction de langue étrangère s'apparente à une tâche de "recréation » et permet selon lui d'éclairer le concept d'interprétation, car « tout traducteur est un interprète » (Gadamer 1976: 233). La tâche du traducteur ne diffère pas, dès lors, de la tâche herméneutique posée par n'importe quel autre texte dans la mesure où tous les objets de l'herméneutique sont en quelque sorte " étrangers ». Traduire ou comprendre, c'est transposer le sens dans le contexte où vit l'interlocuteur, c'est-à-dire l'insérer dans une autre culture. Le comportement du traducteur, comme celui de l'interprète, ressemble à celui qui caractérise toute entente dans la conversation. Le "dialogue herméneutique " - la réciprocité de l'entente dans la conversation - implique selon lui que les partenaires respectent ce qui leur est "étranger et opposé ». Gadamer rappelle que la signification littérale du mot inter-près désigne « d'abord la fonction 
première de l'interprète, qui se tient entre des locuteurs de langues différentes et qui (...) réunit ceux que leurs langues respectives séparaient. Dans ce cas, l'obstacle de la langue étrangère est levé. (...) Le rôle de l'interprète est de lever l'élément étranger qui rend un texte incompréhensible.» (Gadamer 1991: 220) ${ }^{19}$ Pour sa part, Ricœur écrit : «Toute interprétation se propose de vaincre un éloignement, une distance, entre l'époque culturelle révolue à laquelle appartient le texte et l'interprète lui-même. En surmontant cette distance, en se rendant contemporain du texte, l'exégète peut s'approprier le sens : d'étranger il veut le rendre propre, c'est-à-dire le faire sien ; " (Ricœur 1969 : 20). Antoine Berman dans L'Épreuve de l'étranger définit aussi la visée de la traduction en ces termes: "ouvrir au niveau de l'écrit un certain rapport à l'Autre, féconder le Propre par la médiation de l'Étranger» (Berman 1984 : 16). Rappelons que c'est encore Schleiermacher, le premier, qui a su formuler ce thème central de l'herméneutique moderne en évoquant «l'étranger et sa nature médiatrice » (Schleiermacher 1999 : 91).

Le livre de Judith Butler Gender Trouble a paru tardivement en langue française en 2005, quinze ans après sa publication en anglais. Dans un article intitulé en français «Genre et trouble dans le genre ", publié dans le recueil Philosopher en langues. Les intraduisibles en traduction sous la direction de Barbara Cassin (2014), elle explique que «le terme gender a d'abord paru incompréhensible " aux yeux des Français, le mot renvoyant pour eux à la grammaire et aux genres littéraires. Lorsque Gender Trouble fut proposé à une maison d'édition française, nous dit-elle, "l'éditeur déclara que le livre était "inassimilable", telle une substance étrangère ou un immigré indésirable à tenir à bonne distance des frontières françaises " (Butler 2014: 155). On voit comment ce problème de traduction et le malentendu culturel qui lui était lié illustrent l'écart entre l'« étranger » et le " propre » appelant le travail d'interprétation, tel qu'il a été évoqué précédemment par Ricœur, Gadamer ou Berman. Le terme de gender, écrit encore la philosophe, est resté notoirement difficile à traduire dans les autres langues romanes au cours des années 1980 et 1990 (Butler 2014 : 151). En effet, selon les différentes langues, il ne recouvre pas la même signification. Celle-ci peut illustrer un parti pris culturel, mais la définition est également instruite par les débats des féministes au sein même des différentes cultures. Elle raconte ainsi que dans les premières traductions en langue allemande de Gender Trouble, le terme gender avait été traduit par Geschlechtsidentität, ce qui signifiait que l'apparence de genre était amalgamée à l'orientation sexuelle (Butler 2014 : 153).

Dans une conférence bilingue la confrontant ainsi directement à l'exercice de la traduction, présentée à l'Université de Lausanne le 13 novembre 2013 et intitulée «Is Gender (un)translatable ? $»^{20}$, Judith Butler a rappelé que le féminisme dépend de la traduction: sans celle-ci, il n'y a pas de liens possibles pour le féminisme. Comment ceci peut-il influencer la manière dont on raconte l'histoire du féminisme, s'est-elle demandé. Certaines œuvres circulent, et d'autres pas, à l'intérieur d'un cadre hégémonique favorisant certaines langues aux dépens des autres. De surcroit, certains textes demeurent intraduisibles étant donné que leur contenu relève d'un contexte particulier qui les rend indicibles dans d'autres langues. Gender Trouble a été traduit en 27 langues, la traduction donnant lieu à des œuvres multiples qui se détachent de l'intention de l'auteure en ouvrant à la pluralité des interprétations : «there are mine and not mine at the same time " a commenté Judith Butler lors de sa conférence à Lausanne ${ }^{21}$. 
La théoricienne et traductrice féministe Gayatri Spivak, avec qui Judith Butler a signé un livre intitulé L'État global (2007), évoque dans "The Politics of Translation » la traduction comme lecture («translation as reading ») et la lecture comme traduction ("reading as translation»), tel qu'évoqué précédemment par Butler à propos des traductions et interprétations de Gender Trouble. Dans cet essai évoquant explicitement les liens entre traduction et politique, Spivak décrit la traduction comme permettant de donner accès à des textes, notamment en langue anglaise, au plus grand nombre de féministes, avec toutes les implications politiques qui s'ensuivent dans le contexte du féminisme international. La traduction est la lecture la plus attentive qui soit, pour Gayatri Spivak, qui souligne l'importance du geste d'hospitalité, de même que le contexte et l'histoire dans l'acte traduisant, une affirmation qui fait ici écho à la tradition herméneutique, même si elle ne s'en réclame pas (Spivak 2004 [1993] : 370, 371 et 384 ).

\section{La lecture du monde : faire sens dans l'ordre du politique}

\section{" yet women have always survived/dans une autre langue » (Nicole Brossard)}

Pour Barbara Cassin, l'acte de traduire, l'acte de parole et l'acte de langue «sont tous trois des performances, linguistique, langagière, interprétative" (Cassin 2014: 19). S'inscrivant dans l'horizon herméneutique du sens en mettant en avant l'aspect performatif, Jean-René Ladmiral affirme lui aussi que "la traduction est une herméneutique en acte du texte-source » (Ladmiral $2006: 111$, en italique dans le texte). La dimension de l'action est également présente chez Ricœur dans ce qu'il appelle son « entreprise d'herméneutique militante » et la réinscription de sa théorie du texte dans une théorie de l'action (Ricœur 1986 : 8). Le titre de son livre Du texte à l'action souligne la dimension politique des relations entre les êtres humains, le politique étant pour lui étroitement lié à l'éthique autour de la question de la justice. Sa définition de la relation éthique, qu'il explicitera dans Soi-même comme un autre comme étant celle de "la visée de la "vie bonne" avec et pour autrui dans des institutions justes» (Ricœur, 1990 : 202, en italique dans le texte), est en résonance avec la pensée féministe appelant à un changement social et politique.

Dans l'horizon herméneutique contemporain et celui du politique, Charles Taylor affirme que l'objet de ce qu'il appelle une «science interprétative », qu'il applique aux sciences humaines et notamment au champ du politique, « doit pouvoir être décrit en termes de sens et de non-sens, de cohérence et d'incohérence ", et cela est vrai, selon lui, pour la réalité sociale et les significations communes parmi lesquelles nous pourrions inclure la hiérarchie des sexes et l'inégalité dans l'espace social (Taylor 2011 : 81, c'est nous qui soulignons). Professeur de sciences politiques et de philosophie politique, auteur d'une œuvre majeure, Taylor s'est aussi engagé concrètement dans la vie politique canadienne en étant candidat à plusieurs reprises au Québec pour le Nouveau Parti Démocrate aux élections fédérales canadiennes. Sa réflexion philosophique et herméneutique n'est donc pas dissociée du souci de comprendre les problèmes de son époque, sa pensée n'est pas séparée de l'action politique. La question du sens soulevée par Taylor et le problème du malentendu au cœur de la tradition herméneutique 
rejoignent ici la réflexion de l'écrivaine féministe québécoise Nicole Brossard, qui affirme que s'interroger sur "l'émergence d'une culture au féminin dans le contexte des millénaires et au présent d'une société patriarcale» ne s'aurait s'envisager qu'autour de l'expression "faire sens $»^{22}$. La réalité patriarcale, pour les femmes, explique-t-elle, constitue un non-sens, car dans le lieu qu'elle habite, « la femme n'existe pas, c'est-à-dire qu'elle ne fait pas sens $\aleph^{23}$. Florence Rochefort, dans son Histoire mondiale des féminismes, évoque la question de la lisibilité liée à la compréhension: "L'une des tâches des féminismes est de rendre lisibles les inégalités de genre déniées par l'idée d'une supposée nature innée ou prédestinée. » (Rochefort $2018: 4$ )

La lutte des féministes pour l'égalité et la reconnaissance, illustrée notamment par les grands combats de Gisèle Halimi en tant qu'avocate au cours de ses soixante-dix ans au service de la cause des femmes et de la justice, est une réponse fondamentale à la question d'un sens existentiel tel que l'exprime Nicole Brossard citée ci-dessus. Gisèle Halimi raconte dans l'entretien accordé à Annick Cojean publié en 2020 sous le titre Une farouche liberté, que dès l'âge de sept ou huit ans on l'a obligée à la maison à se mettre au service de ses frères. Avant même d'éprouver un sentiment de révolte, cette situation l'a plongée, nous dit-elle, dans « une immense perplexité » qu'elle exprime en termes de non-sens: "Pourquoi cette différence? Elle n'avait aucun fondement ni aucun sens. » (Halimi 2020 : 19-20) Elle a choisi, pour finir, de faire la grève de la faim pour ne pas servir ses frères et lorsque ses parents finirent par céder, ce fut nous ditelle, sa « première victoire féministe » (Halimi $2020: 20$ ).

Si le mot "genre" renvoie à la composante culturelle et au caractère fondamentalement social des distinctions fondées sur le sexe, c'est également une grille de lecture et d'interprétation (on rejoint ici l'herméneutique), une manière de penser le monde et le rapport au pouvoir à travers le prisme de la différence de sexes (cf. Scott 1988). Susanne de Lotbinière-Harwood rappelle en outre le rapport au corps dans toute traduction et le fait que « l'effet du genre » est ressenti par le corps lisant. La question du genre, nous dit-elle, doit être prise en compte dans la réflexion sur la traduction: "Ainsi tout acte de lecture, que ce soit la lecture d'une œuvre ou l'interprétation d'un évènement, est codé au départ. Or, traduire, c'est d'abord lire. » (Lotbinière-Harwood 1991 : 18) Et elle affirme qu'au-delà des mots qui s'incarnent dans la traduction, c'est un corps qui parle. L'histoire de l'émancipation des femmes est inséparable de la lutte pour la réappropriation de leurs corps, du combat pour l'avortement et la répression du viol mené par Gisèle Halimi, la conquête d'une position de sujet et le refus d'être traitée simplement comme objet. De la maîtrise de la reproduction de la femme à l'avortement, le corps de la femme a été un enjeu politique par excellence et, selon Geneviève Fraisse, "un écran où chacun projette sa violence ", qu'elle soit physique, symbolique ou morale (Fraisse 2020: 96-103) ${ }^{24}$. La violence domestique ou sociale, rappelle encore Catherine Malabou, qui représente l'un des symptômes de la domination masculine, est également une violence théorique, car la femme n'a jamais pu se définir autrement «que par la violence qui lui est faite " (Malabou 2009: 115). Cette culture de la violence à l'égard des femmes, illustrée plus récemment par l'ampleur du mouvement \# $\mathrm{MeToo}^{25}$, nous ramène à l'éthique en lien avec la question de l'hégémonie dans l'acte du traduire qui, selon Ben Van Wyke, traverse toute l'histoire de la traduction: " the history of translation studies has been, for the most part, also the history of translation ethics" (Van Wyke 2013: 548). Le refus du paradigme dominant, rappelle-t-il, est associé aux études sur le genre, comme aux études postcoloniales, ainsi qu'aux travaux des théoriciennes féministes de la traduction (Van 
Wyke 2013: 552) ${ }^{26}$. Pour Susanne de Lotbinière-Harwood, la traduction est une " éthique au féminin » qui prend en compte l'expérience des femmes et en particulier celle de leur corps (Lotbinière-Harwood de 1991 : 72-73).

\section{L'invisibilité de la traduction comme celle des femmes}

Le concept et la catégorie de "genre ", analysant l'organisation sociale de la relation entre les sexes et la répartition du pouvoir qui en résulte, ont fait leur apparition dans le champ de la traductologie à la fin des années 1980. Depuis lors, de nombreux livres et articles ont été publiés sur la question dans le monde entier, mais citons en particulier l'article précurseur de Lori Chamberlain « Gender and the Metaphorics of Translation » (1988), ainsi que les ouvrages Gender in Translation de Sherry Simon (1996) et Translation and Gender de Luise von Flotow (1997). Ces deux théoriciennes canadiennes ont été de véritables pionnières dans ce domaine, comme l'ont été les traductrices féministes au Québec, parmi lesquelles Susanne de Lotbinière-Harwood ${ }^{27}$.

La rencontre de la traductologie et de la pensée féministe autour de la question de la langue et celle de l'autorité s'est révélée particulièrement fructueuse en ouvrant de nouvelles perspectives théoriques et pratiques, notamment en rapport avec l'éthique, la question de la visibilité des femmes et celle de leur reconnaissance. On se souvient du cri des féministes dans les années 1970: "La libération des femmes passe par le langage. » En traduction, comme dans les études de genre, il est largement admis que le langage façonne le sens : il ne reflète pas seulement la réalité, il contribue à la créer. Plus largement, le champ féministe vise à un changement de représentation. Sherry Simon rappelle que la traductologie et la pensée féministe ont pris leur essor à une époque qui a accordé un rôle prépondérant au langage: "Both feminism and translation are concerned by the way 'secondariness' comes to be defined and canonized; both are tools for a critical understanding of difference as it is represented in language. » (Simon 1996 : 8) On ne saurait évoquer, plus généralement, la question de l'invisibilité en traduction sans mentionner aussi le livre de Lawrence Venuti, The Translator's Invisibility (1995), qui examine de manière approfondie l'herméneutique de Schleiermacher en vue de proposer une éthique de la traduction qui refuserait le paradigme hégémonique dominant et qui souligne la portée politique d'un choix traductologique.

Les études sur le genre en traduction, en particulier sur les métaphores sexistes et sexuelles, ont dévoilé les rapports de pouvoir qui sous-tendent toute activité de traduction et le symbolisme de l'ordre patriarcal à l'œuvre dans les textes et leur traduction ${ }^{28}$. Si la métaphore du vêtement ou du costume a souvent été utilisée pour décrire la traduction, on pourrait avancer que les analyses sur les métaphores sexistes et sexuelles ont contribué à déshabiller le patriarcat ${ }^{29}$. Lori Chamberlain a ainsi démontré comment les métaphores utilisées pour décrire la traduction et les discours sexualisés qui lui sont associés illustrent l'idéologie du patriarcat et contribuent à la reproduction des normes patriarcales (Chamberlain 2004 [1988]) : 306-321). Dans une perspective gadamérienne, on pourrait ajouter que ces analyses ont révélé le fonctionnement du patriarcat comme illustrant de faux plutôt que de vrais "préjugés ». Par ailleurs, rappelons que si Ricœur a d'abord défini l'herméneutique comme l'interprétation des textes et des symboles, il en a élargi ensuite la définition à l'interprétation des métaphores (Ricœur 2000). Il est désormais largement admis que 
les métaphores ont non seulement une fonction cognitive (Lakoff et Johnson 1980), mais qu'elles représentent un soubassement de la pensée en donnant forme et sens à la réalité et en structurant un monde (Blumenberg 2006).

Revenons à la célèbre métaphore des "belles infidèles", attribuée au grammairien Gilles Ménage (1630-1692), qui a fait florès dans l'histoire de la traduction et l'imaginaire occidental. Susanne de Lotbinière-Harwood affirme « que face au langage, la femme se trouve au même niveau d'infériorité, voire d'illégitimité, que la traduction face à l'œuvre d'origine » (Lotbinière-Harwood 1991 : 21-22). Elle reprend l'expression "les belles infidèles", nous dit-elle, pour "décrire la position subversive » qu'elle adopte " en traduisant au féminin » : "Si les "belles" du XVIIe étaient "infidèles" aux œuvres d'origine au profit de leurs propres priorités, les "re-belles" du XXe siècle sont infidèles à la loi du langage patriarcal en ce qu'il nous interdit, nous, les femmes. » (Lotbinière-Harwood 1991 : 21) La traduction a le plus souvent été confinée à un rôle subalterne, à la passivité et à la subordination associées au féminin dénoncées par Susanne de Lotbinière-Harwood, par rapport à l'autorité de l'écrivain, identifiée au principe créateur masculin. Au cours de l'histoire, nombre de femmes écrivaines qui ne pouvaient faire valoir leurs compétences et affirmer leur autorité de créatrices, se livrèrent à une activité de traduction. La romancière britannique George Eliot, de son vrai nom Mary Anne Evans (1819-1880) et qui fut obligée d'adopter un pseudonyme masculin, s'est d'abord fait connaître comme traductrice de Ludwig Feuerbach. On reconnaît aujourd'hui que cette grande figure de la littérature anglaise a permis d'introduire dans le roman un niveau de complexité psychologique inédit avec une approche d'ordre éthique, notamment dans The Mill on the Floss (1860) ou Middlemarch (1872). Luise von Flotow soutient que l'on n'a pas seulement redécouvert des auteures « disparues », mais également des traductrices « disparues » (Von Flotow 1998 : 123).

L'historienne Michelle Perrot raconte dans Le chemin des femmes combien la voix des femmes a été empêchée au cours de l'histoire et leurs écrits détruits. On songe à Mme de Staël dans sa lutte contre Napoléon et dont la censure impériale en 1810 ordonna la mise au pilon de son livre De l'Allemagne, qui prônait l'idéal du cosmopolitisme en proposant un modèle étranger ${ }^{30}$. Dans un essai intitulé De l'esprit des traductions, écrit au cours de son second voyage en Italie et publié en 1816, Mme de Staël dénoncera l'école française du XVIIIe siècle des "belles infidèles ». D'une part, elle est contre l'annexion de l'impérialisme français en traduction dans l'ordre esthétique, et d'autre part, contre le régime de Bonaparte en politique, pour les mêmes raisons éthiques, à savoir la reconnaissance de l'altérité. Le rapport du "propre » à l'« étranger » est illustré de manière exemplaire, pour elle, dans l'art de la traduction et le geste d'hospitalité. Dans Dix années d'exil, elle écrira que l'Empereur s'efforcera de l'« annuler » par l'exil qui lui était imposé, contribuant à son exclusion du champ de la pensée et de l'action politiques, comme du domaine de la littérature. Geneviève Fraisse avance qu'au-delà du harcèlement, il existe une autre forme de jouissance du pouvoir (et qui caractérisait notamment Bonaparte) consistant «dans la disqualification systématique de la femme $»^{31}$ - ce dont elle a fait l'expérience elle-même, notamment dans son travail intellectuel - et dont fut victime Mme de Staël, calomniée de son vivant en tant que femme, et que l'Empereur s'efforcera en vain de réduire au silence. 


\section{L'éthique de l'« hospitalité langagière »}

30 Nicole Brossard, évoquée précédemment, qui vit au Québec et dont le parcours est imprégné de son militantisme pour la cause féministe, a fait de la traduction le thème principal et l'enjeu essentiel de son roman Le Désert Mauve (Brossard 1987). Le roman en entier est en français et nous donne à lire une traduction du français en français. Que pourrait donc signifier ici une traduction d'une langue dans la même langue? Dans un poème intitulé "Sociologie", elle écrit: "yet women have always survived/dans une autre langue » (Brossard 1989: 43, en italique dans le texte). Les femmes parlent la langue étrangère du patriarcat qui les habite, nous dit-elle, « avec un accent » et ce qui caractérise les personnes qui parlent avec un accent, c'est de déformer les sons, ce qui peut créer des malentendus ${ }^{32}$. Susanne de Lotbinière-Harwood, qui a assuré la traduction anglaise du roman Le Désert Mauve (Mauve Desert), écrit dans Re-Belle et infidèle. La traduction comme pratique de réécriture au féminin : "Toutes les femmes sont bilingues. Nous "possédons" forcément la langue dominante, de fabrication masculine, puisque c'est la seule qui a une valeur reconnue. » Par contre, nous dit-elle encore, les femmes communiquent entre elles dans une langue de tradition orale et au niveau du langage corporel: «Traduire nous est donc une pratique familière.» (LotbinièreHarwood de $1991: 13)$

31 En évoquant la question de la pluralité des langues, Ricœur nous dit que l'idée ne serait pas de créer un espéranto, mais d'être polyglotte, «c'est-à-dire de pratiquer, d'habiter plusieurs maisons de langue " $^{33}$. Il s'agit là du modèle de l'« hospitalité langagière ", qu'il met en avant dans «Le paradigme de la traduction » et également dans une contribution à une réflexion commune sur la construction européenne intitulée "Quel éthos nouveau pour l'Europe?» (Ricœur 2004/2016: 43/29, 1992). En choisissant ici de mettre en jeu le concept herméneutique de médiation dans le champ de «l'imaginaire social », il présente trois modèles d'intégration liés à "l'identité » et l'«altérité » : la traduction, l'échange des mémoires et le pardon. «La traduction, » écrit-il, « est la seule manière de manifester l'universalité du langage dans la dispersion des langues », ce qui laisse entendre selon lui une pluralité humaine indépassable (Ricœur 1992 : 115).

Dans le grand entretien qu'il accorda à Jean Daniel en 1988, le fondateur du Nouvel Observateur et le philosophe évoquèrent "l'étrangeté de l'étranger ", Ricœur définissant le modèle de l'hospitalité langagière dans sa dimension politique : « Recevoir la langue étrangère chez soi, et habiter la langue de l'autre.» Et il ajoutait: «Politiquement, c'est très important, cela entraîne le droit d'être reçu dans tout pays, non comme l'ennemi mais comme un ami parce que justement l'hospitalité n'annule pas la différence. $»^{34}$ Si Ricœur se montre sensible à l'idée de réciprocité et à la reconnaissance d'autrui en écho aux origines étymologiques de l'hospitalité en Occident liées à des notions de pouvoir et d'égalisation, il évoque également dans cet entretien les rapports de domination: "Le principe d'hospitalité est égalisant. La domination est hiérarchisante. » Et il explique encore: "Nous traînons le boulet de l'autorité : (...) mais nous n'arrivons pas à la faire sortir du simple vouloir vivre ensemble, qui serait égalisant et conduirait, s'il était seul, à l'utopie de l'égalité absolue de toutes les cultures. $»^{35}$ (Daniel 1998) Dans une perspective féministe, le modèle de l'hospitalité langagière que Ricœur nous invite à transposer " en termes d'imagination " et "d'organisation politique " (Ricœur 1992: 107), pourrait ainsi s'élargir à «l'utopie » (l'une des expressions de l'imaginaire social) de la « traduction d'une langue 
culturelle dans une autre» (Ricœur 1992: 116): le décentrement de la culture patriarcale et de ses représentations laisserait dès lors place à la langue et à la réalité des femmes. Il s'agirait là de la "relation horizontale du vouloir vivre ensemble " auquel nous invite la traduction dans le geste d'hospitalité (Ricœur 1992 : 116).

Dans "Le paradigme de la traduction", Ricœur place ses réflexions sous l'égide de George Steiner («comprendre, c'est traduire») et L'épreuve de l'étranger d'Antoine Berman. La traduction est la rencontre avec l'étranger ou l'altérité, écrit-il, en posant « un problème éthique» (Ricœur 2004: 21-22,42; 2016: 13,29). Berman, dans La Traduction et la lettre ou l'Auberge du lointain, écrit que la traduction "appartient originairement à la dimension éthique " par sa visée de fidélité. Il définit alors l'éthique en ces termes en renvoyant à Totalité et infini d'Emmanuel Levinas: "L'acte éthique consiste à reconnaître et à recevoir l'Autre en tant qu'Autre. » (Berman 1999 : 74-75, en italique dans le texte) Dans la pensée de Levinas, ce rapport implique que l'on ne se saisisse pas de l'autre; il est éthique parce qu'il est non-violent. Au contraire, la visée d'appropriation et d'annexion d'autrui, qui caractérise ce que Bermann appelle la traduction ethnocentrique, rapporte tout à sa propre culture et considère l'Étranger de manière négative « ou tout juste bon à être annexé, adapté, pour accroître la richesse de cette culture " (Bermann 1999: 29). C'est la conception de la traduction que l'on associe en France aux «belles infidèles» des XVIIe et XVIIIe siècle, évoquée précédemment dans le contexte des métaphores sexistes et sexuelles, et dénoncée par Mme de Staël dans son essai sur les traductions. La «captation du sens » dont parle Berman dans le contexte de la traduction ethnocentrique et annexioniste pourrait alors aussi s'appliquer au régime patriarcal décrit précédemment par Nicole Brossard, où « la femme n'existe pas, c'est-à-dire qu'elle ne fait pas sens $»^{36}$. Pour Berman, au contraire, la traduction se doit d'être décentrement, ouverture, dialogue et "mise en rapport " (Berman 1984 : 16). La captation de sens représente aussi, selon lui, la négation de l'histoire de Babel (Berman 1999: 34). Ce mythe de Babel, dont «le paradigme de la traduction » autorise une relecture positive pour Ricœur - évoquant dans ce contexte Walter Benjamin, Hannah Arendt et Antoine Berman avec «le désir de traduire »nous appelle à la traduction comme "une tâche, non au sens d'une obligation contraignante, mais au sens de la chose à faire pour que l'action humaine puisse simplement continuer » (Ricœur, $2004: 38,36 ; 2016: 26,24$, en italique dans le texte). La traduction représente dès lors pour Ricœur un "pari », qui s'inscrit dans une conception de l'universel comportant l'acceptation «de la différence indépassable entre le propre et l'étranger » (Ricœur $2004: 11,42 ; 2016: 4,29$ ).

L'historien et anthropologue Jean-Pierre Vernant rappelle la symbolique des origines de l'hospitalité en Occident, qui engage le rapport du dehors et du dedans dans le monde hellénique représenté par Hermès et Hestia. Si Hestia est la déesse de l'espace domestique, Hermès est le messager, l'ambassadeur, « un voyageur qui vient de loin et qui déjà s'apprête au départ » (Vernant 1963 : 14). Elle est au centre de la maison, lieu emblématique de l'hospitalité, et lui, sur le pas de la porte protégeant le seuil. L'espace est aussi psychique : dans le monde gréco-romain, traverser un territoire géographique implique de franchir un espace d'altérité. Hermès siège à l'entrée des maisons et des villes, il est là sur les tombeaux, présent aux carrefours : c'est le maître des routes et le guide des voyageurs dans cette vie et jusque vers l'Hadès (ibid.). L'hospitalité comporte pour le voyageur, l'étranger venant de l'extérieur et qui n'a pas de place ni de statut 
dans la cité, de franchir le seuil et d'être admis à l'intérieur, de passer de Hermès à Hestia, à savoir de l'inégalité de statut pour devenir l'égal de l'hôte.

Si le féminisme peut se comprendre comme la volonté de refuser le paradigme archaïque et « l'idéologie » représentée par la domination masculine, selon l'opposition formulée par Ricœur, "l'utopie» à laquelle il nous invite est "un exercice de l'imagination pour penser autrement ", sa fonction de contestation étant «toujours de proposer une société alternative» (Ricœur $1984: 61)^{37}$. L'invitation à étendre le geste éthique de l'hospitalité linguistique au rapport entre les cultures elles-mêmes - avec l'art du transfert dans l'univers mental d'une autre culture - s'appliquerait ainsi à celle des femmes, en tenant compte de leurs croyances, de leurs convictions et de l'ensemble de ce que Ricœur nomme les « repères de sens " (Ricœur $1992: 109)$. Le "paradigme de la traduction " pourrait dès lors, au même titre que "l'utopie " dans sa fonction libératrice, renouveler le champ de l'imaginaire social et politique.

\section{BIBLIOGRAPHIE}

BAKER, Mona. 1998. « Translation Studies » in The Routledge Encyclopedia of Translation Studies, BAKER, Mona (éd.). Londres et New York : Routledge, 277-280.

BERMAN, Antoine. 1984. L'épreuve de l'étranger. Culture et traduction dans l'Allemagne romantique. Paris : Éditions Gallimard.

BERMAN, Antoine. 1989. « La traduction et ses discours » META vol. 34, $\mathrm{n}^{\circ}$ 4, 672-679.

BERMAN, Antoine. 1999. La Traduction et la lettre ou l'Auberge du lointain. Paris : Éditions du Seuil. BLUMENBERG, Hans. 2006. Paradigmes pour une métaphorologie. Traduction de Didier Gammelin. Paris : Librairie Philosophique J. Vrin.

BOUVERESSE, Jacques. 1991. Herméneutique et linguistique. Combat : Éditions de l'éclat.

BROSSARD, Nicole. 1987. Le Désert mauve. Montréal : Éditions de l'Hexagone.

BROSSARD, Nicole. 1989. Installations (avec et sans pronoms). Trois-Rivières, Québec : Les Écrits des Forges.

BROSSARD, Nicole. 2007. « De radical à intégrales » Sisyphe [En ligne], consulté le 30 avril 2020. URL : http://sisyphe.org/spip.php?article2252.

BUTLER, Judith. 2006. Gender Trouble: feminism and the subversion of identity. New York : Routledge.

BUTLER, Judith et SPIVAK, G.C. 2007. L'État global. Traduction de Françoise Bouillot. Paris : Éditions Payot.

BUTLER, Judith. 2013. « Théorie du genre : Judith Butler répond à ses détracteurs ", Nouvel Observateur [En ligne], consulté le 20 décembre 2020. URL : https://bibliobs.nouvelobs.com/ essais/20131213.OBS9493/theorie-du-genre-judith-butler-repond-a-ses-detracteurs.html 
BUTLER ; Judith. 2014. « Genre et trouble dans le genre », traduction Hélène Quiniou, in Philosopher en langues. Les intraduisibles en traduction, CASSIN, Barbara (dir.). Paris : Éditions Rue d'Ulm/Presses de l'École normale supérieure, 149-165.

CASSIN, Barbara (dir.). 2014. Philosopher en langues. Les intraduisibles en traduction. Paris : Éditions Rue d'Ulm/Presses de l'École normale supérieure.

CHAMBERLAIN, Lori. 2004 [1988]. « Gender and the Metaphorics of Translation » in The Translation Studies Reader, VENUTI, Lawrence (éd). Londres et New York : Routledge, 2e édition, 306-321

CHESTERMAN, Andrew. 1997. Memes of Translation. Amsterdam/Philadelphia : John Benjamins Publishing Company.

CIXOUS, Hélène. 2010. Le Rire de la Méduse et autres ironies. Paris : Éditions Galilée.

CIXOUS, Hélène. 2020. « Hélène Cixous : "Je suis née solidaire des femmes" » L'Obs [En ligne], consulté le 2 janvier 2021. URL : https://www.nouvelobs.com/idees/20201227.OBS38081/helenecixous-je-suis-nee-solidaire-des-femmes.html

CODE, Lorraine. 2003. Feminist Interpretations of Hans-Georg Gadamer. University Park : The Pennsylvania State University Press.

DANIEL, Jean. 1998. «Les grands entretiens de Jean Daniel : Paul Ricœur » Nouvel Observateur [En ligne], consulté le 30 avril 2020. URL : https://www.nouvelobs.com/jean-daniel/ 20200227.OBS25353/les-grands-entretiens-de-jean-daniel-paul-ric-ur-7-8.html

DELAGE, Pauline. 2021. « Haro sur les violences sexuelles !» Sciences Humaines n 63 [En ligne], consulté le 13 juillet 2021. URL : https://www.scienceshumaines.com/haro-sur-les-violencessexuelles_fr_43507.html

FOUCAULT, Michel. 1966. Les mots et le choses. Paris : Éditions Gallimard.

FRAISSE, Geneviève. 2017. Entretien « Le corps de la femme est un écran où chacun projette sa violence » i-D.vice [En ligne], consulté le 11 juillet 2021. URL : https://i-d.vice.com/fr/article/ xwygz4/le-corps-de-la-femme-est-un-ecran-ou-chacun-projette-sa-violence

FRAISSE, Geneviève. 2020. Féminisme et philosophie. Paris : Éditions Gallimard.

GADAMER, Hans-Georg. 1975. «L'herméneutique philosophique » Studies in Religion/Sciences religieuses, vol. $5, \mathrm{n}^{\circ} 1,3-13$.

GADAMER, Hans-Georg. 1976. Vérité et méthode. Les grandes lignes d'une herméneutique philosophique. Traduction d'Étienne Sacre, révision de Paul Ricœur. Paris : Éditions du Seuil.

GADAMER, Hans-Georg. 1991. « Texte et interprétation » in L'art de comprendre. Ecrits II. Herméneutique et Champ de l'expérience humaine. Textes réunis par Pierre Fruchon et traduits par Isabelle Julien-Deygout, Philippe Forget, Pierre Fruchon, Jean Grondin et Jacques Schouwey. Paris : Éditions Aubier.

GÉAL, François. Trésor des métaphores de la traduction (TMT) [En ligne], consulté le 30 avril 2020. URL : http://recherche.univ-lyon2.fr/tmt/

GILLIGAN, Carol et Naomi SNIDER. 2019. Pourquoi le patriarcat? Traduction de Cécile Roche revue par Vanessa Nurock. Paris : Climats.

GRONDIN, Jean. 1987. « Herméneutique et relativisme » Communio n XII.5 (sept.-oct. 1987) : 101-120. 
GRONDIN, Jean. 1993. L'universalité de l'herméneutique. Préface de Hans-Georg Gadamer. Paris : Presses Universitaires de France.

GRONDIN, Jean. 1993. L'horizon herméneutique de la pensée contemporaine. Paris : Librairie Philosophique J. Vrin.

HALIMI, Gisèle. 1994. «Établir l'égalité politique : un référendum pour les femmes » Le Monde diplomatique [En ligne], consulté le 27 janvier 2021. URL : https://www.monde-diplomatique.fr/ 1994/10/HALIMI/7505.

HALIMI, Gisèle avec Annick COJEAN. 2020. Une farouche liberté. Paris : Bernard Grasset.

HALSEMA, Annemie et HENRIQUES, Fernanda (éds.). 2016. Feminist Explorations of Paul Ricœur's Philosophy. New York et Londres : Lexington Books.

HECKMAN, Susan. 2003. « The Ontology of Change: Gadamer and Feminism » in Feminist Interpretations of Hans-Georg Gadamer. University Park : The Pennsylvania State University Press, 181-201.

HERITIER, Françoise. 2002. Masculin/Féminin II. Dissoudre la hiérarchie. Paris : Odile Jacob.

ILLOUZ, Eva. 2020. «Les femmes exigent une profonde transformation “par le bas", fait sans précédent dans l'histoire » Le Monde [En ligne], consulté le 2 février 2021. URL : https:// www.lemonde.fr/idees/article/2020/10/16/eva-illouz-les-femmes-exigent-une-profondetransformation-par-le-bas-fait-sans-precedent-dans-1-histoire_6056199_3232.html

JEANNERET, Michel. 2015. « Hermès est terminé, mais l'interprétation est infinie » Fabula-LhT [En ligne], consulté le 30 avril 2020. URL : http:www.fabula.org/lht/14/jeanneret.html

JERVOLINO, Domenico. 2004. « La question de l'unité de l'œuvre de Ricœur à la lumière de ses derniers développements. Le paradigme de la traduction » Archives de Philosophie t. 67, 659-668.

KAFKA, Franz. 1945. La Colonie pénitentiaire. Nouvelles suivies d'un Journal intime. Traduction et préface de Jean Starobinski. Paris : Egloff ; Fribourg : Librairie de l'Université de Fribourg.

LADMIRAL, Jean-René. 2006. «L'empire des sens » in Le sens en traduction, LEDERER, Marianne (éd.). Paris : Lettres modernes Minard, 109-125.

LADMIRAL, Jean-René. 2015. Sourcier ou cibliste. Les profondeurs de la traduction. Paris : Les Belles Lettres (coll. « Traductologiques »), 2e édition, revue.

LAKOFF, George et JOHNSON, Mark. 1980. Metaphors We Live By. Chicago : University of Chicago Press.

LOTBINIERE-HARWOOD, Susanne de. 1991. Re-belle et infidèle. La traduction comme pratique de réécriture au féminin. The Body Bilingual. Translation as a Rewriting in the Feminine. Montréal : les éditions du remue-ménage/Women's Press.

MALABOU, Catherine. 2009. Changer de différence. Le féminin et la question philosophique. Paris : Éditions Galilée.

MOLINO, Jean. 1985. « Pour une histoire de l'interprétation : les étapes de l'herméneutique » Philosophiques vol. 12, $\mathrm{n}^{\circ}$ 1, 73-103.

NAVARRE, Maud. 2021. « La force d'une idée » Sciences Humaines n 63 [En ligne], consulté le 13 juillet 2021. URL : https://www.scienceshumaines.com/la-force-d-une-idee_fr_43475.html

NAVES, Marie-Cécile. 2020. La démocratie féministe. Paris : Calmann-Lévy.

OUAKNIN, Marc-Alain. 2020. Émission de France-Culture « L'art est la matière » de Jean de Loisy le 26 janvier 2020 intitulée « La Genèse en miroir de la peinture moderne », consulté le 28 janvier 
2021. URL : https://www.franceculture.fr/emissions/lart-est-la-matiere/la-genese-en-miroir-dela-peinture-moderne-0

OUAKNIN, Marc-Alain. 2019. «"L'atelier du traduire”. Quelques remarques sur une nouvelle traduction de la Bible » Tsafon, Revue d'études juives du Nord 77/2019, 105-124 [En ligne], consulté le 28 janvier 2021. URL : https://doi.org/10.4000/tsafon.1913

RADIER, Véronique. 2019. «Carol Gilligan et Michelle Perrot déshabillent le patriarcat » L'Obs n 2872, 69-73, [En ligne], consulté le 27 janvier 2021. URL : https://www.nouvelobs.com/idees/ 20191124.OBS21498/carole-gilligan-et-michelle-perrot-deshabillent-le-patriarcat.html RICÆEUR, Paul. 1966. « Prévision économique et choix éthique » Esprit, n 346(2), 173-193. RICCEUR, Paul. 1969. Le conflit des interprétations. Paris : Éditions du Seuil. RICEUR, Paul. 1986. Du texte à l'action. Essais d'herméneutique, II. Paris : Éditions du Seuil. RICCEUR, Paul. 1990. Soi-même comme un autre. Paris : Éditions du Seuil.

RICCEUR, Paul. 1992. «Quel éthos nouveau pour l'Europe? » in Imaginer l'Europe. Le marché intérieur européen, tâche culturelle et économique, KOSLOWSKI, P. (dir.). Paris : Cerf, 107-116.

RICEUR, Paul. 2000. «"Quo Vadis ?” Un entretien avec Paul Ricœur par Yvanka B. Raynova » Labyrinth vol. 2 [En ligne], consulté le 27 janvier 2021. URL : http://phaidon.philo.at/ iaf/ Labyrinth/2000/ricoeur.html

RICEUUR, Paul. 2004 [1999]. « Le paradigme de la traduction », in Sur la traduction. Paris : Bayard.

RICÆEUR, Paul. 2016. Sur la traduction, Paris : Les Belles Lettres (coll. « Traductologiques ».

ROCHEFORT, Florence. 2018. Histoire mondiale des féminismes. Paris : Que sais-je ?

SCHLEIERMACHER, Friedrich. 1987. Herméneutique. Traduction et édition Christian Berner. Paris : Le Cerf.

SCHLEIERMACHER, Friedrich. 1999. Des différentes méthodes du traduire. Traduction Antoine Berman. Paris : Éditions du Seuil.

SCOTT, Joan W. 1988. « Genre : une catégorie utile d'analyse historique » Les Cahiers du GRIF n 37-38, 125-153.

SCOTT-BAUMANN, Alison. 2016. « Speak to Silence and Identify Absence on Campus: Sister Prudence and Paul Ricœur on the Negated Woman Question » in Feminist Explorations of Paul Ricœur's Philosophy, HALSEMA, Annemie et HENRIQUES, Fernanda (éds.). New York et Londres : Lexington Books, 37-61.

SIMON, Sherry. 1996. Gender in Translation. Cultural identity and the politics of transmission. Londres et New York : Routledge.

SPIVAK, Gayatri Chakravorty. 2004 [1993]. « The Politics of Translation » in Outside in the Teaching Machine. New York et Londres, Routledge : 179-200. Réédité dans VENUTI, Lawrence (éd.), The Translation Studies Reader, 2e édition. Londres : Routledge, 369-388.

STAROBINSKI, Jean. 1987. «L'Art de Comprendre », Avant-propos à L'Herméneutique de Friedrich Schleiermacher. Genève : Labor et Fides, 5-11.

STAROBINSKI, Jean. 2016. La beauté du monde. Edition établie sous la direction de Martin RUEFF. Paris : Quarto Gallimard.

ST. ANDRÉ, James. 2010. Thinking through Translation with Metaphors. Manchester, U.K. et Kinderhook, NY, USA : St. Jerome Publishing. 
TAYLOR, Charles. 2011. «L'interprétation et les sciences de l'homme » in Herméneutique contemporaine. Comprendre, interpréter, connaître. Textes réunis par Denis THOUARD. Paris : Librairie Philosophique J. Vrin, 79-111.

TAYLOR, Charles. 1997. La liberté des modernes. Paris : Presses Universitaires de France.

VAN WYKE, Ben. 2010. «Imitating Bodies and Clothes. Refashioning the Western Conception of Translation » in Thinking through Translation with Metaphors, James St. ANDRÉ (éd.). Manchester, U.K. et Kinderhook, NY, USA : St. Jerome Publishing, 17-46.

VAN WYKE, Ben. 2013. «Translation and ethics » in The Routledge Handbook of Translation Studies, Carmen MILLAN et Francesca BARTRINA (éds.). New York : Routledge : 548-560.

VENUTI, Lawrence. 1991. « Genealogies of Translation Theory: Schleiermacher » TTR (Traduction, terminologie, rédaction) vol. $4, \mathrm{n}^{\circ} 2,125-150$.

VENUTI, Lawrence. 1995. The Translator's Invisibility. A History of Translation. Londres et New York : Routledge.

VERNANT, Jean-Pierre. 1963. «Hestia-Hermès. Sur l'expression religieuse de l'espace et du mouvement chez les Grecs » in L'Homme t. 3, n 3, 12-50 [En ligne], consulté le 27 janvier 2021. URL : https://www.persee.fr/doc/hom_0439-4216_1963_num_3_3_366578.

VON FLOTOW, Luise. 1998. « Le féminisme en traduction », Palimpsestes $n^{\circ} 11$ intitulé « Traduire la culture ». Paris : Presses de la Sorbonne Nouvelle, 117-133.

WARNKE, Georgia. 2013. « Feminism and Hermeneutics » Hypatia vol. 8, n 1, 81-98.

WILHELM, Jane. 2004. « La traduction, principe de perfectibilité, chez Mme de Staël » META vol. $49, \mathrm{n}^{\circ} 3,692-705$.

WILHELM, Jane. 2014. « Anthropologie des lectures féministes de la traduction » TTR (Traduction, terminologie, rédaction) $27, \mathrm{n}^{\circ}$ 1,149-188.

WILHELM, Jane. 2019. « Des métaphores de genre en traduction. Vers un nouvel "imaginaire social” » de genere [En ligne], consulté le 30 avril 2020. URL : http://www.degenere-journal.it/

WOLTON, Dominique. 2019. « Ouverture », Hermès 83. Les stéréotypes, encore et toujours. Paris : CNRS Editions, 12-19.

\section{NOTES}

1. Le mot "féminisme " apparaît tardivement en français sous la plume d'Alexandre Dumas fils, qui l'aurait utilisé en 1872 pour railler les femmes luttant pour l'égalité des droits. Il fut ensuite adopté dans son sens militant par la suffragiste française Hubertine Auclert (1848-1914), puis il sera repris plus largement en France avant de se diffuser dans d'autres langues. ( $C f$. Rochefort $2018: 7)$

2. Voir le Trésor des métaphores de la traduction (TMT), une base de données lancée par François Géal en 2014, Université Lyon 2 (http://recherche.univ-lyon2.fr/tmt/). Cf. le recueil édité par James St. André intitulé Thinking through Translation with Metaphors (2010).

3. Paul Ricœur, «Le paradigme de la traduction », in Sur la traduction, Paris, Bayard, 2004, p. 27 et p. 40, en italique dans le texte. Réédition revue : Sur la traduction, Paris, Les Belles Lettres (coll. «Traductologiques »), 2016, p. 17 et p. 27. Comme la première édition est épuisée depuis quelque dix ans, nous indiquerons les deux paginations.

4. Cf. Hans-Georg Gadamer, 1976, Vérité et méthode. Les grandes lignes d'une herméneutique philosophique, Paris : Éditions du Seuil, p. 149. 
5. Cf. Mona Baker, l'article intitulé «Translation studies » du Routledge Encyclopedia of Translation Studies (1998: 279).

6. Dans «L'Avertissement » à Jean Starobinski. La beauté du monde, Martin Rueff le qualifie de "plus grand herméneute littéraire de langue française du XXe siècle » (2016:11). Starobinski fut aussi le premier traducteur de Franz Kafka en langue française : traduction et présentation de $L a$ Colonie pénitentiaire, nouvelles suivies d'un Journal intime en 1945.

7. Cf. Lawrence Venuti « Genealogies of Translation Theory: Schleiermacher » (1991: 125-150).

8. L'Ars Critica ou « l'ensemble d'opérations qui permettent de séparer le vrai du faux, de porter un jugement décisif sur un texte ou sur un évènement ", selon Jean Molino, et qui conduira à la critique philologique et historique du XIXe siècle, est avant tout critique d'authenticité des textes («Pour une histoire de l'interprétation: les étapes de l'herméneutique » Philosophiques vol. 12, $\mathrm{n}^{\circ}$ $1:$ 73-103, p. 90).

9. Cf. Marc-Alain Ouaknin, "L'atelier du traduire". Quelques remarques sur une nouvelle traduction de la Bible ", Tsafon, Revue d'études juives du Nord 77/2019, 105-124 (https://doi.org/ 10.4000/tsafon.1913).

10. Cf. Michel Jeanneret, "Hermès est terminé, mais l'interprétation est infinie » Fabula-LhT 14, «Pourquoi l'interprétation?», février 2015 (https://www.fabula.org/lht/14/jeanneret.html)

11. Hans-Georg Gadamer, "L'herméneutique philosophique ", Sciences religieuses/Studies in religion (1975 :12). Dans l'article intitulé « Texte et interprétation » de L'art de comprendre. Ecrits II, Gadamer écrit: "Même dans le domaine des sciences de la nature, le fondement de la connaissance ne peut éluder cette conséquence herméneutique selon laquelle le "donné” n'est pas dissociable de l'interprétation. » (1991 : 206). Cf. Jean Grondin, L'universalité de l'herméneutique, préface de Hans-Georg Gadamer (1993).

12. Jean Grondin, dans un article intitulé "Herméneutique et relativisme », Communio ${ }^{\circ}$ XII.5, écrit : «Ignorer la structure préalable de la précompréhension, c'est se couper de la condition minimale de l'objectivité. Car celui qui se prononce dénué de préjugés s'expose d'autant plus aveuglement à leur empire. » $(1987: 114)$.

13. Récemment traduit dans la revue GLAD! : https://journals.openedition.org/glad/2057

14. $C f$. Judith Butler, "Genre et trouble dans le genre ", traduction Hélène Quiniou, in Barbara Cassin (dir.), Philosopher en langues. Les intraduisibles en traduction (2014:149,151 notamment).

15. Von Humboldt, auquel se réfère Barbara Cassin, tout comme Ricœur, parle plutôt de diversité (Cf. Ricœur $2004: 53 ; 2016: 37$ ).

16. https://www.nouvelobs.com/idees/20201227.0BS38081/helene-cixous-je-suis-nee-solidairedes-femmes.html

17. Paul Ricœur, Sur la traduction (2004: 44-45) et dans l'édition revue des Belles Lettres (2016: 30). Cf. Jacques Bouveresse, Herméneutique et linguistique (1991:39).

18. Cf. Barbara Cassin: "La traduction est en effet doublement vectorisée : comme toute interprétation, elle part du "fait de la non-compréhension" (le Faktum de l'herméneutique de Schleiermacher) et ce, sous condition "d'un amour simple et sans prétention pour l'original" (Humboldt).» (2014, p. 17)

19. Cf. Ricœur dans Le conflit des interprétations (1969: 8).

20. https://www.unil.ch/fdi/files/live/sites/fdi/files/shared/Journee_13nov2013.pdf

21. Il y aurait un parallèle à établir avec «Le Rire de la Méduse » (1975) d'Hélène Cixous, devenu manifeste mondial traduit dans toutes les langues du monde, y compris en férö̈en. Cette dernière parle de toutes les «métamorphoses» de «la Méduse», qui pour elle était «un texte de guérilla», mais qui, dit-elle encore, «a tellement voyagé, elle a connu tellement de métamorphoses, qu'à la fin nous sommes réconciliées ». (https://www.nouvelobs.com/idees/ 20201227.OBS38081/helene-cixous-je-suis-nee-solidaire-des-femmes.html)

22. En italique dans le texte. Nicole Brossard, «De radical à intégrales ", http://sisyphe.org/ spip.php?article2252, en italique dans le texte. "De radical à intégrales » est le texte d'une 
conférence donnée à l'occasion du colloque "L'émergence d'une culture au féminin » à l'Université de Montréal, au Québec, en 1982.

23. http://sisyphe.org/spip.php?article2252

24. Voir également l'entretien de Geneviève Fraisse avec Micha Barban Dangerfield, i-D.vice, 4 avril 2017. https://i-d.vice.com/fr/article/xwygz4/le-corps-de-la-femme-est-un-ecran-ouchacun-projette-sa-violence

25. Cf. Geneviève Fraisse, Féminisme et philosophie (2020, en particulier le chapitre V intitulé «L'affaire Weinstein est une révolte historique et politique »: 120-125). Voir également « Haro sur les violences sexuelles !» in La grande histoire du féminisme. La force d'une idée (2021 : 68-71).

26. Ben Van Wyke écrit à ce propos : «While there is certainly not one specific notion of ethics associated with these tendencies, they stem from the belief that language, far from being neutral and natural as it has historically been presented, has typically served the interests of powerful entities that have used it to, in the examples above, for instance, subordinate women and colonial subjects to men and colonial powers, respectively, thus (mis)representing the former by assimilating them into discourse of the latter and concealing alternative realities. » (Van Wyke $2013: 552)$

27. A ce titre, Luise von Flotow a été invitée à présenter la conférence inaugurale du colloque international Traduire, un engagement politique ? le 29 novembre 2018 à l'Université Paris Diderot. Sa contribution s'intitulait "The challenges of 'Global English' for transnational feminist translation and translation studies ».

28. Cf. Jane Wilhelm, « Anthropologie des lectures féministes de la traduction » (2014: 149-188); et Jane Wilhelm, " Des métaphores de genre en traduction. Vers un nouvel "imaginaire social" " de genere (http://www.degenere-journal.it/). Antoine Berman souligne combien "les définitions conceptuelles de la traduction sont rares " et que celle-ci ne peut être «définie " que par des métaphores (1999: 43), d'où l'importance de l'analyse du discours métaphorique dans une perspective féministe.

29. Nous reprenons ici le titre d'un article paru dans l'obs : "Gilligan et Perrot déshabillent le patriarcat » $\left(n^{\circ} 2872,21 / 11 / 2019\right.$, pp. 69-73). Pour ce qui concerne la métaphore du vêtement, voir le "Trésor des métaphores de la traduction", Université Lumière Lyon 2 (http:// recherche.univ-lyon2.fr/tmt/) et l'article de Ben Van Wyke intitulé «Imitating Bodies and Clothes. Refashioning the Western Conception of Translation » dans le recueil de

James St. André (dir.), Thinking through Translation with Metaphors (2010, pp. 17-46).

30. $C f$. Jane Wilhelm, «La traduction, principe de perfectibilité, chez Mme de Staël » (2004, pp. 692-705).

31. Geneviève Fraisse préfère le terme de « disqualification » pour redéfinir le mot de « sexisme » (2020, p. 110).

32. http://sisyphe.org/spip.php?article2252

33. https://www.nouvelobs.com/jean-daniel/20200227.OBS25353/les-grands-entretiens-de-jeandaniel-paul-ric-ur-7-8.html

34. Ibid.

35. Ibid.

36. http://sisyphe.org/spip.php?article2252

37. Ricœur écrit aussi les lignes suivantes qui pourraient s'appliquer au combat des féministes : «Il semble, en effet, que nous ayons toujours besoin de l'utopie dans sa fonction fondamentale de contestation et de projection dans un ailleurs radical, pour mener à bien une critique également radicale des idéologies. » (1984: 63) 


\section{RÉSUMÉS}

Nous examinerons dans cet article les lieux de convergence entre traduction, herméneutique et féminisme en invoquant plus particulièrement le «paradigme de la traduction » de Paul Ricœur et son concept d'hospitalité langagière. L'herméneutique philosophique, dans sa dimension politique, nous permet de reconnaître le caractère partial de notre compréhension et de corroborer les arguments féministes relatifs à la dimension interprétative de la différence. Pour Ricœur, la traduction pose un problème éthique et il nous invite aussi à transposer le modèle de l'hospitalité linguistique en termes d'imagination politique. Le paradigme de la traduction pourrait dès lors s'élargir à la traduction de la culture patriarcale et de ses représentations sexistes, parmi lesquelles les métaphores sexuelles, pour laisser place par le décentrement à la langue et à la réalité des femmes dans un vouloir-vivre ensemble qui serait égalisant.

We will examine in this paper the meeting points of translation, hermeneutics and feminism, invoking in particular Paul Ricœur's "paradigm of translation" and his concept of linguistic hospitality. Philosophical hermeneutics, in its political dimension, allows us to acknowledge the partiality of our understanding and to support feminist arguments regarding the interpretative dimension of difference. Translation, for Ricœur, poses an ethical problem and he also invites us to transpose the model of linguistic hospitality in terms of political imagination. The paradigm of translation could consequently expand to the translation of patriarchal culture and its sexist representations, among which sexual metaphors, by its decentering to allow for the language and the reality of women while living together in equality.

\section{INDEX}

Thèmes : Recherches

Keywords : feminism, hermeneutics, interpretation, paradigm of translation

Mots-clés : féminisme, herméneutique, interprétation, paradigme de la traduction

\section{AUTEUR}

\section{JANE ELISABETH WILHELM}

Chercheuse associée au Laboratoire d'études de genre et de sexualité (LEGS), UMR 8238, CNRS/

Université Paris 8 et Université Paris Nanterre. 